\title{
Base subsets of the Hilbert Grassmannian
}

\author{
Mark Pankov
}

go back

\section{Abstract}

Let $H$ be a separable Hilbert space. We consider the Hilbert Grassmannian $\mathcal{G}_{\infty}(H)$ consisting of closed subspaces having infinite dimension and codimension and show that every bijective transformation of $\mathcal{G}_{\infty}(H)$ preserving the class of base subsets is induced by an element of $\mathrm{GL}(H)$ or it is the composition of the transformation induced by an element of $\mathrm{GL}(H)$ and the bijection sending a subspace to its orthogonal complement.

Keywords: Hilbert Grassmannian, base subset, infinite-dimensional topological projective space

MSC 2000: 46C05, 51E24

\section{Introduction}

We start from the classical Grassmannian $\mathcal{G}_{k}(V)$ consisting of all $k$-dimensional subspaces of an $n$-dimensional vector space $V$. A base subset of $\mathcal{G}_{k}(V)$ is the set of $\left(\begin{array}{l}n \\ k\end{array}\right)$ distinct $k$-dimensional subspaces spanned by vectors of a certain base of $V$. This construction is closely related with Tits buildings [5]: the Grassmannians $\mathcal{G}_{k}(V)(k=1, \ldots, n-1)$ are the shadow spaces of the building associated with the group $\mathrm{GL}(V)$ and their base subsets are the shadows of the corresponding apartments. It was proved in [2] that transformations of $\mathcal{G}_{k}(V)$ preserving the class of base subsets are induced by a semilinear isomorphism of $V$ to itself or to $V^{*}$ (the second possibility can be realized only for the case when $n=2 k$ ); a more general result can be found in [3].

Suppose that $V$ is an infinite-dimensional vector space and $\operatorname{dim} V=\aleph$. The group GL $(V)$ acts on the set $\mathcal{S}$ of all subspaces of $V$ and the orbits of this action are called Grassmannians. There are the following three types of Grassmannians:

$$
\mathcal{G}_{\alpha}(V):=\{S \in \mathcal{S} \mid \operatorname{dim} S=\alpha, \operatorname{codim} S=\aleph\}
$$




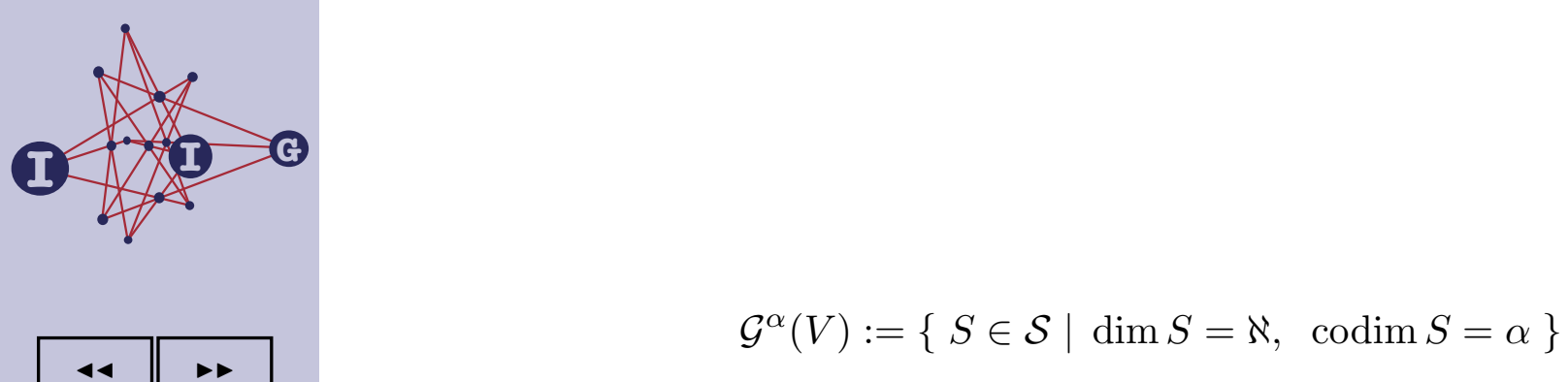

for any cardinality $\alpha<\aleph$ and

$$
\mathcal{G}_{\aleph}(V):=\{S \in \mathcal{S} \mid \operatorname{dim} S=\operatorname{codim} S=\aleph\} .
$$

page $2 / 6$

go back

full screen

close

quit

As in the finite-dimensional case, we define base subsets of Grassmannians. It was shown in [4] that every bijective transformation of $\mathcal{G}_{\alpha}(V), \alpha<\aleph$ is induced by a semilinear automorphism of $V$. The methods of [4] can not be applied to other Grassmannians; this is related with the fact that $V$ and $V^{*}$ have different dimensions and the duality principles do not hold for infinitedimensional vector spaces.

Now suppose that $V$ is a topological vector space. Denote by $\Pi_{V}$ the projective space associated with $V$. The topology of $V$ induces a topology on $\Pi_{V}$ and we talk about an infinity-dimensional topological projective space. A collineation of $\Pi_{V}$ to itself will be called closed if it preserves the class of closed subspaces. It follows from Mackey's results [1] that every closed collineation of $\Pi_{V}$ to itself is induced by an invertible bounded linear operator if $V$ is a real normed space. In the general case, closed collineations are not determined.

In the present note we give a geometrical characterization of closed collineations of the projective space over a separable Hilbert space.

\section{Result}

Let $H$ be a separable Hilbert space (real or complex). We write $\mathcal{G}_{\infty}(H)$ for the Hilbert Grassmannian consisting of all closed subspaces with infinite dimension and codimension. Let $B=\left\{x_{i}\right\}_{i \in \mathbb{N}}$ be a base of $H$ (possibly non-orthogonal). The set of all elements of $\mathcal{G}_{\infty}(H)$ spanned by subsets of $B$ is called the base subset of $\mathcal{G}_{\infty}(H)$ associated with (or defined by) the base $B$.

Every closed collineation of $\Pi_{H}$ to itself induces a bijective transformation of $\mathcal{G}_{\infty}(H)$ preserving the class of base subsets. The bijection

$$
\circ: \mathcal{G}_{\infty}(H) \rightarrow \mathcal{G}_{\infty}(H)
$$

sending subspaces to their orthogonal complements also preserves the class of base subsets.

Theorem 2.1. If $f$ is a bijective transformation of $\mathcal{G}_{\infty}(H)$ preserving the class of base subsets, then $f$ is induced by a closed collineation of $\Pi_{H}$ to itself or it is the composition of the transformation $\circ$ and the transformation induced by a closed collineation of $\Pi_{H}$ to itself. 


\section{Preliminaries}

Let $B=\left\{x_{i}\right\}_{i \in \mathbb{N}}$ be base of $H$, and let $\mathcal{B}$ be the associated base subset of $\mathcal{G}_{\infty}(H)$. We denote by $P_{i}$ the 1 -dimensional subspace containing $x_{i}$ and write $\mathcal{B}_{+i}$ and $\mathcal{B}_{-i}$ for the sets of all elements of $\mathcal{B}$ which contain $P_{i}$ or do not contain $P_{i}$, respectively. Then $\mathcal{B}_{-i}$ consists of all elements of $\mathcal{B}$ contained in the hyperplane

$$
S_{i}:=\overline{B \backslash\left\{x_{i}\right\}}
$$

full screen

close

quit

We say that $\mathcal{X} \subset \mathcal{B}$ is an exact subset if there is only one base subset of $\mathcal{G}_{\infty}(H)$ containing $\mathcal{X}$; otherwise, $\mathcal{X}$ is said to be inexact.

Remark 3.1. It is trivial that $\mathcal{X}$ is exact if and only if for each $i \in \mathbb{N}$ the intersection of all $U \in \mathcal{X}$ containing $P_{i}$ coincides with $P_{i}$. For example,

$$
\mathcal{R}_{i j}:=\left(\mathcal{B}_{+i} \cap \mathcal{B}_{+j}\right) \cup \mathcal{B}_{-i} i \neq j
$$

is an inexact subset. Indeed, the intersection of all elements containing $P_{k}$ coincides with $P_{k}$ if $k \neq i$, however for $k=i$ this intersection is $P_{i}+P_{j}$. Every element of $\mathcal{B} \backslash \mathcal{R}_{i j}$ intersects $P_{i}+P_{j}$ by $P_{i}$. This means that

$$
\mathcal{R}_{i j} \cup\{U\}
$$

is exact and the inexact subset $\mathcal{R}_{i j}$ is maximal. Conversely, every maximal inexact subset of $\mathcal{B}$ coincides with certain $\mathcal{R}_{i j}$ (the proof of this fact is similar to the proof of Lemma 1 in [4]).

Let $\mathcal{B}^{\prime}$ be the base subset of $\mathcal{G}_{\infty}(H)$ defined by a base $B^{\prime}=\left\{x_{i}^{\prime}\right\}_{i \in \mathbb{N}}$. We write $\mathcal{B}_{+i}^{\prime}$ and $\mathcal{B}_{-i}^{\prime}$ for the sets of all elements of $\mathcal{B}^{\prime}$ which contain $P_{i}^{\prime}$ or do not contain $P_{i}^{\prime}$ (respectively); here $P_{i}^{\prime}$ is the 1 -dimensional subspace containing $x_{i}^{\prime}$. We also define

$$
S_{i}^{\prime}:=\overline{B^{\prime} \backslash\left\{x_{i}^{\prime}\right\}}
$$

A bijection $g: \mathcal{B} \rightarrow \mathcal{B}^{\prime}$ is called special if $g$ and $g^{-1}$ map inexact subsets to inexact subsets.

Lemma 3.2. If $g: \mathcal{B} \rightarrow \mathcal{B}^{\prime}$ is a special bijection then there exists a bijective transformation $\delta: \mathbb{N} \rightarrow \mathbb{N}$ such that

$$
g\left(\mathcal{B}_{+i}\right)=\mathcal{B}_{+\delta(i)}^{\prime}, \quad g\left(\mathcal{B}_{-i}\right)=\mathcal{B}_{-\delta(i)}^{\prime} \quad \forall i \in \mathbb{N}
$$

or

$$
g\left(\mathcal{B}_{+i}\right)=\mathcal{B}_{-\delta(i)}^{\prime}, \quad g\left(\mathcal{B}_{-i}\right)=\mathcal{B}_{+\delta(i)}^{\prime} \quad \forall i \in \mathbb{N} .
$$

Proof. This is similar to the proof of Lemma 3 in [4]. 



\section{Proof of the theorem}

Let $f$ be a bijective transformation of $\mathcal{G}_{\infty}(H)$ preserving the class of base subsets. We consider an arbitrary base subset $\mathcal{B} \subset \mathcal{G}_{\infty}(H)$. Then $\mathcal{B}^{\prime}:=f(\mathcal{B})$ is a base subset and $\left.f\right|_{\mathcal{B}}: \mathcal{B} \rightarrow \mathcal{B}^{\prime}$ is a special bijection. Suppose that $B=$ $\left\{x_{i}\right\}_{i \in \mathbb{N}}$ and $B^{\prime}=\left\{x_{i}^{\prime}\right\}_{i \in \mathbb{N}}$ are bases associated with $\mathcal{B}$ and $\mathcal{B}^{\prime}$, respectively. Let $P_{i}, P_{i}^{\prime}, S_{i}, S_{i}^{\prime}$ and $\delta: \mathbb{N} \rightarrow \mathbb{N}$ be as in the previous section. It is clear that we can assume that $\delta$ is identical. We have to consider the following possibilities:

(A) $\left.f\right|_{\mathcal{B}}$ is a special bijection of first type,

(B) $\left.f\right|_{\mathcal{B}}$ is a special bijection of second type.

Case (A). First we claim that

$$
f\left(\left[P_{i}\right]\right)=\left[P_{i}^{\prime}\right] \quad \forall i \in \mathbb{N} .
$$

Let $U, M, N$ be as in Lemma 3.4. By Lemma 3.3,

$$
f(M) \cap f(N)=P_{i}^{\prime}, \operatorname{codim} \overline{f(M)+f(N)}>1 .
$$

If $\widetilde{\mathcal{B}}$ is a base subset containing $U, M, N$ then (1) together with Lemma 3.3 show that the restriction of $f$ to $\widetilde{\mathcal{B}}$ is a special bijection of first type. Bases associated with $\widetilde{\mathcal{B}}$ and its $f$-image contain vectors lying in $P_{i}$ and $P_{i}^{\prime}$ (respectively), and Lemma 3.2 implies that $f(U)$ belongs to $\left[P_{i}^{\prime}\right]$. Thus $f\left(\left[P_{i}\right]\right) \subset\left[P_{i}^{\prime}\right]$. Since $f^{-1}$ preserves the class of base subsets, we can also prove the inverse inclusion. The claim follows.

Using Lemma 3.5 we show that

$$
f\left(\left[S_{i}\right]\right)=\left[S_{i}^{\prime}\right] \quad \forall i \in \mathbb{N} .
$$

For any 1-dimensional subspace $P$ there exists $S_{i}$ which do not contain $P$. We consider a base $\widetilde{B}$ of $H$ such that each vector of $\widetilde{B}$ is contained in $S_{i}$ or $P$. The latter equality guarantees that the restriction of $f$ to the associated base subset is a special bijection of first type, and arguments given above imply the existence of a 1-dimensional subspace $P^{\prime}$ such that $f([P])=\left[P^{\prime}\right]$.

Similarly, for every closed hyperplane $S$ we choose $P_{i} \notin S$ and establish the existence of a closed hyperplane $S^{\prime}$ such that $f([S])=\left[S^{\prime}\right]$.

Therefore, $f$ gives a closed collineation of $\Pi_{H}$. This collineation induces $f$.

Case (B). Since $\left.\circ f\right|_{\mathcal{B}}$ is a special bijection of first type, of is induced by a closed collineation. 


\section{Acknowledgment}

The result of this paper was presented on 32. Arbeitstagung über Geometrie und Algebra (9 - 11 February, 2006, Hamburg). The author thanks the organizers (A. Blunck, H. Kiechle, A. Kreuzer, H.-J. Samaga, H. Wefelscheid) for the invitation and financial support.

\section{References}

[1] G. W. Mackey, Isomorphisms of normed linear spaces, Ann. of Math. 43 (1942), 244-260.

[2] M. Pankov, Transformations of Grassmannians and automorphisms of classical groups, J. Geom. 75 (2002), 132-150.

[3] _ A characterization of geometrical mappings of Grassmann spaces, Results Math. 45 (2004), 319-327.

[4] _ Base subsets of Grassmannians: infinite-dimensional case, European J. Combin. 28 (2007), 26-32.

[5] J. Tits, Buildings of spherical type and finite BN-pairs, Lect. Notes in Math. 386, Springer-Verlag, 1974.

\section{Mark Pankov}

Department of Mathematics and Information Technology, University of WARMia AND MAZURY, Żolnierska 14A,, 10-561 OlszTYN,, Poland

e-mail: pankov@matman.uwm.edu.pl 